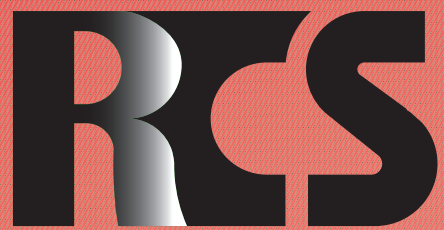

Depósito legal ppi $201502 Z U 4662$

Esta publicación científica en formato digital es continuidad de la revista impresa Depósito Legal: pp $197402 Z U 789$

- ISSN: 1315-9518 • ISSN-E: 2477-9431

Revista de Ciencias Sociales

Universidad del Zulia. Revista de la Facultad de Ciencias Económicas y Sociales Vol. XXVII. No. 2

Abril-Junio 2021

Esta publicación científica en formato digital es continuidad de la revista impresa Depósito Legal: pp $197402 Z$ Z789 ISSN: 1315-9518 

de la población estudiantil afrodescendiente: Experiencia de un colectivo de estudiantes

\title{
Inclusión social de la población estudiantil afrodescendiente: Experiencia de un colectivo de estudiantes universitarios
}

\author{
Granja Escobar, Luis Carlos*
}

\section{Resumen}

La educación, como derecho fundamental cumple una función importante para el ascenso social, sobre todo, para aquellas poblaciones que por diversas razones de inequidad son identificadas como vulnerables. El presente artículo sintetiza los resultados de investigación realizada a un colectivo afrodescediente de estudiantes que desarrolla diversas actividades (cine-foros, celebración del día de la afrocolombianidad, entre otros) para generar bienestar e identidad étnica a los mismos estudiantes de la Universidad Santiago de Cali en Colombia, con el objetivo de evidenciar el papel que cumplen estas organizaciones en la inclusión social en la educación superior. La investigación se plantea siguiendo los fundamentos epistemológicos del enfoque socio-crítico, descriptivo. La metodología utilizada es predominantemente cualitativa, aunque, se utilizan instrumentos de orden cuantitativo; donde mediante encuestas a la población estudiantil afrodescendiente y entrevista colectiva a los integrantes del Centro de Estudios Afrocolombianos, se logra como resultado determinar la importancia que para ellos ha tenido la organización en sus vidas universitarias. Se concluye, que las organizaciones afrodescendientes estudiantiles pueden realizar aportes importantes en la construcción de una educación inclusiva, dada la importante función que cumplen como protectoras y generadoras de relaciones sociales para las personas discriminadas.

Palabras clave: Inclusión; afrodescendientes; educación; colectivo de estudiantes; Universidad.

\footnotetext{
Doctorando en Ciencias Sociales y Jurídicas de la Universidad Rey Juan Carlos, España. Docente Tiempo Completo de la Universidad Santiago de Cali, Colombia. E-mail: 1c.granja.2019@alumnos.urjc.es (iD ORCID: https://orcid. org/0000-0002-1546-1542
} 


\title{
Social inclusion of the Afro-descendant student population: Experience of a group of university students
}

\begin{abstract}
Education, as a fundamental right, fulfills an important function for social advancement, especially for those populations that for various reasons of inequity are identified as vulnerable. This article synthesizes the results of research carried out on an Afro-descendant group of students that develops various activities (cinema-forums, celebration of Afro-Colombian Day, among others) to generate well-being and ethnic identity to the same students of the Santiago de Cali University in Colombia, with the aim of demonstrating the role that these organizations play in social inclusion in higher education. The study is descriptive. The methodology used is predominantly qualitative, although quantitative instruments are used; where, through surveys of the Afro-descendant student population and a collective interview with the members of the Center for Afro-Colombian Studies, it is possible to determine the importance that the organization has had for them in their university lives. It is concluded that Afro-descendant student organizations can make important contributions in the construction of an inclusive education, given the important role that they fulfill as protectors and generators of social relations for discriminated people..
\end{abstract}

Keywords: Inclusion; afro-descendants; education; students collective; University.

\section{Introducción}

La educación, como derecho fundamental cumple una función importante para el ascenso social, sobre todo, para aquellas poblaciones que por diversas razones de inequidad son identificadas como vulnerables. Así, la educación se convierte en la principal estrategia para transformar las realidades de pobreza, discriminación y desigualdades sociales. De allí, la importancia que tienen las instituciones de educación superior, particularmente, las universidades en el desarrollo de conocimiento, habilidades y competencias necesarias para la vida personal y profesional (Martin-Fiorino, 2020).

En tal sentido, el presente artículo busca evidenciar el papel que pueden cumplir las organizaciones estudiantiles universitarias para, la inclusión social y cultural, evitando con ello la deserción de estudiantes afrodescendientes en su formación profesional. Lo anterior, tiene importancia en la medida que, Colombia y la ciudad de Cali, se caracterizan por ser uno de los países y ciudades latinoamericanas con mayor población afrodescendiente. No obstante, es el grupo étnico con los más bajos indicadores de bienestar social (Barbary y Urrea, 2004; Rodríguez, Alfonso y Cavelier, 2008).

En cuanto a la educación, los indicadores evidencian que la población afrodescendiente es la que menos años promedio tienen en educación, con mayor analfabetismo, lo cual significa que, son personas con menos oportunidades para graduarse en todos los niveles educativos, si se las compara con las demás etnias. En la siguiente Tabla 1, se puede ilustrar dicha situación. 


\section{Tabla 1}

Indicadores de bienestar de la población afrocolombiana comparada con las otras etnias

\begin{tabular}{llcc}
\hline & & Afrocolombiano & No Afrocolombiano \\
\hline Educación & Analfabetismo & $11,7 \%$ & $7,0 \%$ \\
& $\begin{array}{l}\text { Años promedio de educación } \\
\text { (hombres) }\end{array}$ & $6,9 \%$ & $8,1 \%$ \\
& $\begin{array}{l}\text { Años promedio de educación } \\
\text { (mujeres) }\end{array}$ & $6,4 \%$ & $8,2 \%$ \\
\hline Pobreza & NBI & $53,7 \%$ & $42,2 \%$ \\
Mercado & Pobreza & $9,5 \%$ & $7,4 \%$ \\
laboral & Tasa de desempleo & $6,3 \%$ & $3,4 \%$ \\
Deslazamiento & Intensidad desplazamiento (promedio & $40,4 \%$ & $44,3 \%$ \\
& 2000-2002) & $6,31 \%$ & $3,42 \%$ \\
Salud & Migración por violencia & $6,78 \%$ & $3,74 \%$ \\
\hline
\end{tabular}

Fuente: Elaboración propia, 2020 con base en Ministerio de Cultura y Universidad de los Andes 2009.

Está claro que la educación es un factor clave para disminuir la pobreza y la inequidad

social (Organización de Naciones Unidas [ONU], 2020), en la medida que se potencializa el desarrollo y bienestar, en este caso, de la población afrodescendiente. Por lo anterior, se debe procurar colocar en marcha medidas que permitan la permanencia de la población afrodescendiente en el sistema educativo. Al respecto, la Red Universitaria Mutis (2015) menciona que:

Las menores tasas de alfabetismo y asistencia escolar conducen a logros educativos inferiores para afrocolombianos e indígenas, que presentan porcentajes mayores de personas sin educación y educación primaria, con respecto al resto de la población. Además, muestran porcentajes ostensiblemente inferiores en la educación superior frente al resto de la población. De hecho, los resultados del censo de 2005 muestran que, en el ámbito nacional, el porcentaje de población indígena con educación superior alcanzo el 2,4\%, aproximadamente, la población afrodescendiente el $8 \%$ y para la población blanca-mestiza fue
$12,6 \%$. Esto significa que la tasa de participación en la educación superior es aproximadamente 4 veces más alta para los blancos o mestizos que para los indígenas, y 0,5 veces más alta para los afro descendientes que para las personas blancas o mestizas. (p.15)

Particularmente, para la educación superior, la tendencia que la población afrodescendiente sea una de la de menos logros, en cuanto al nivel educativo, se reafirma con el Censo Nacional de Población y Vivienda realizado en el 2018, en el cual los resultados indican que el $14,3 \%$ de la población afrodescendiente han alcanzado un nivel educativo superior, entre tanto la media nacional está en un 18,8\% (Departamento Administrativo Nacional de Estadística [DANE], 2019).

Existen diversas explicaciones, que pretenden ilustrar las causas de la baja participación de la población afrodescendiente en la educación superior. Entre ellas, la discriminación racial, que inciden para que algunas personas decidan abandonar sus sueños de ser profesionales; la adaptación cultural, la cual afecta a las personas que 
provienen de municipios muy pequeños o distantes, que conservan formas de ver la vida y prácticas culturales muy diferentes a las dinámicas de las grandes urbes en donde están situadas las universidades (Red Universitaria Mutis, 2015).

También, existen factores relacionados con los bajos resultados en las pruebas de admisión a las Universidades Estatales y en los Exámenes de Estado, que se aplican una vez se culmina la educación secundaria. Los resultados de dicho examen, es el principal requerimiento para acceder a las universidades del Estado. Todo lo anterior, es consecuencia de la baja calidad de la educación que recibieron en primaria y secundaria en los departamentos habitados mayormente por población afrocolombiana (Red Universitaria Mutis, 2015).

La reflexión al respecto, conlleva a pensar que la inclusión social en la educación debe entenderse más allá, de atender la población en situación de discapacidad; para plantaerla -también- desde una perspectiva cultural. Lo cual implique, para las instituciones -desde su reponsabilidad social de la educación- una mayor promoción de las políticas que, amplíen la cobertura educativa (Núñez, et al., 2019). La importancia de la inclusión social es que, ella es uno de los factores de mayor impacto en el desarrollo personal y en la construcción de una sociedad más justa y equitativa (Rubio, Estrada y Pedraza, 2020).

Así las cosas, preguntarse por el papel que, cumplen las organizaciones afrodescendientes al interior de las instituciones de educación superior, resulta relevante para encontrar alternativas posibles que, permitan una mayor inclusion social de dicha población, aumentando su identidad cultural, pertenencia étnica y la permanencia en el sistema educativo. En consecuencia, con lo anterior, el objetivo principal de este articulo, es determinar el papel que cumplen estas organizaciones en la inclusión social en el ambito educativo, tomando como caso de estudio la experiencia de los integrantes del colectivo del Centro de Estudios Afrocolombianos (CEAFRO) de la Universidad Santiago de Cali en Colombia.

\section{Algunos aspectos teorícos: Bases para comprender la importancia del colectivo de CEAFRO}

Como se mencionó anteriormente, las posibilidades de la población afrodescendiente para formarse como profesionales en las universidades, son reducidas si se las compara con el resto de la población Colombiana. En el marco de dicha problemática, que a demás es histórica; surgen las políticas de inclusión, (también llamadas acciones afirmativas) como forma para atender la situación. Ejemplos de algunas decisiones legislativas que pueden tomarse en calidad de políticas de inclusión a nivel nacional, en torno, a la educación para los afrodescendientes, son:

a. Ley 70 de 1993: Ley que aborda el reconocimiento de las comunidades negras como grupo étnico. Establece en el artículo 40, que el gobierno central realizará reservas presupuestales para garantizar mayores oportunidades de ingreso a la Educación Superior. Además, de crearse el fondo especial de becas destinado a estudiantes afrodescendientes, para educación superior.

b. Decreto 2249 de 1995: Decreto por el cual se conforma la Comisión Pedagógica de Comunidades Negras.

c. Decreto 1627 de 1996: Decreto por el cual se crea el fondo de créditos educativos, administrados por el ICETEX ${ }^{(1)}$, con el objetivo de apoyar a los estudiantes afrodescendientes de bajo recursos.

d. Decreto 1122 de 1998: Describe la Cátedra de Estudios Afrocolombianos.

Adicional, a lo anterior, se encuentra que en los decretos y lineamientos, que orientan y definen las condiciones necesarias, para el otorgamiento de los registros calificados de los programas académicos y, para que un programa sea considerado de alta calidad, se debe cumplir con algunas exigencias de inclusión de la población en situación de vulnerabilidad, como es el caso de la población afrodescendiente. A manera de ilustración, se tiene que en los lineamientos 
para la acreditación de programas de pregrado, publicados por el Consejo Nacional de Acreditación (CNA, 2013), mencionan en los aspectos a evaluar de las características $31 \mathrm{y}$ 32, respectivamente, las siguientes exigencias a verificar: "Estrategias que permitan a los estudiantes vincularse a redes de apoyo orientadas a contrarrestar las situaciones de vulnerabilidad" (p.43); así como, "Registros periódicos de la caracterización de los estudiantes teniendo en cuenta variables de vulnerabilidad" (p.44). Así las cosas, las instituciones de educación superior, en su interior, deben:

Responder positivamente a las demandas sociales para la democratización radical de la universidad, poniendo fin a una historia de exclusión de grupos sociales y de sus saberes, en lo que ha sido protagonista la universidad durante mucho tiempo, desde antes de la actual fase de globalización capitalista. (De Sousa, 2007, p.52)

En consonancia con lo anterior, en esta busqueda de la inclusión de los grupos menos favorecidos, se debe considerar los aspectos culturales. Al respecto, De Sousa (2007), mencionará lo siguiente:

En los paises pluriétnicos y multinacionales, el conocimiento pluriuniversitario está emergiendo aún del interior de la propia universidad cuando estudiantes de grupos minoritarios (étnicos u otros) entran en la universidad y verifican que su inclusión es una forma de exclusión: se enfrentan con la tábula rasa que está hecha a partir de sus culturas y de los conocimientos propios de las comunidades de donde son originarios. (p.45)

Enesesentido, lainclusiondelapoblacion afrodescendiente, puede considerarse como una necesidad educativa; por lo menos, asi lo reconoce la Organización para la Cooperación y el Desarrollo Económicos (OCDE, 2011), al considerar las deventajas sociales y condiciones de marginalidad que afecta en una mayor proporcion a dicha población etnica. Señala que todo proceso de inclusión no debe conformarse con la insercion de los estudiantes al sistema educativo superior, sino que también, debe garantizar su permanencia con acompañamiento pertinente para atender los riesgos de abandono al mismo. Para ello, debe fomentar actividades, como por ejemplo, los grupos de ayuda o de apoyo mutuo (García, Fletcher y Romero, 2009).

De otro lado, abordando de forma teórica la dinámica de los colectivos y/o grupos de personas, desde la perspectiva de la interacción (Shaw, 2013), se retoma la importancia que tiene para la constitución y dinámica colectiva; las formas como se relacionan unos con otros. La idea de que existe algo en común entre los miembros, ya sea solucionar una problemática que los aqueja o alcanzar una meta, permite comprender el sentido y valor que los individuos le dan al colectivo.

Por su parte, la perspectiva del campo (Lewin, 1978), aporta un aspecto importante que no se recoge desde la anterior teoría y es precisamente, tomar en cuenta al individuo desde sus necesidades, sentimientos y metas personales. Particularmente, la identidad que es considerada como una necesidad básica de todo ser humano (Chenet, Arévalo y Palma, 2017) pues, ella hace que el individuo se relacione de una forma u otra con el colectivo. En pocas palabras, la construcción socio histórica de los individuos, es importante tenerla en cuenta en el análisis de los grupos. Un aporte relevante de la perspectiva del individuo es que, desde su condición de ser humano producen su realidad social.

Dicho de otro modo, toda realidad objetual es fruto de la actividad objetivadora del sujeto. Todos los seres humanos, sean o no profesionales de las ciencias sociales, producen su realidad social. De manera transductiva (esto es inventado a partir de lo dado) construimos la realidad al tiempo que nos modificamos (hablando con propiedad, nos autoconstruimos). Realidad, que, de manera recursiva, está al servicio de las prácticas que diariamente realizamos. (Montañés, 2007, p.19)

La idea de Montañés (2007), brinda elementos para comprender que, los sujetos sociales se relacionan desde sus visiones y significados del mundo. Las cuales se configuran conforme a la praxis social, en consonancia con las experiencias vividas. Por 
otra parte, la perspectiva de los sistemas ofrece varios aspectos para tener en cuenta. Entre ellos, las diferenciaciones que propone analizar en las interacciones entre los miembros de un grupo: Por estatus, acceso a recursos, control sobre las personas y la identificación con el grupo, resultan aspectos importantes para explicar las problemáticas internas.

De los aspectos comunes y que pueden ser considerados como edificantes en el análisis de las problemáticas de los colectivos, conforme a lo recorrido hasta el momento, son varios: La participación y las formas de participación de los individuos; la identidad colectiva; y, la relación entre los intereses y metas individuales con las colectivas.

Adicional a las anteriores consideraciones, es importante mencionar que, las relaciones que se entretejen en las organizaciones sociales, al estructurarse a partir de relaciones dialógicas, suponen una peculiar dinámica entre participación y compromiso, en la cual el elemento socio-afectivo juega un papel fundamental, generando así mismo formas de identificación basadas más en el compromiso, que promueve la imitación de comportamientos de entrega, apego y profundos sentimientos de pertenencia (Montero, 2003). Todos los anteriores elementos, son los que algunos autores han denominado lo constitutivo de la cultura organizacional.

Lo primero que hay que tener en cuenta al abordar una probable, pero siempre discutida definición de cultura, conforme a la corriente teórica y posiciones ontológicas, es que parece ser una situación social compleja y nada fácil de definir (Diez,1999). Sin embargo, con base a los elementos expuestos, se podría admitir las siguientes dos definiciones de cultura, enfocadas hacia la organización: La cultura como fenómeno social, se produce y se adquiere a través de las relaciones sociales presentes entre los miembros de un grupo, dicha cultura determina el modo de comportarse en distintas situaciones (Diez, 1999). En complementariedad, un sistema cultural se entenderá como la integración de creencias y valores, que comparten las personas de la organización, y que se manifiestan por múltiples formas (Fernández, 2009).

Otro concepto central para tener en cuenta -inclusive en el abordaje del término de cultura organizacional- es el de conciencia, definido como la captación individual o grupal de la ideología imperante (Montero, 2003), y que puede ser: Sumisa y práctica, crítica-integradora o crítica-liberadora. La importancia del concepto de conciencia, reside en el hecho que es sobre este donde se asienta la relación de poder, puesto que se necesita que el agente que carece de acceso al recurso construya la situación como desigual, con la consiguiente insatisfacción. Es decir, es necesario que el agente sienta una necesidad y tenga aspiraciones (Montero, 2003).

Las anteriores referencias teóricas, fueron utilizadas para comprender la experiencia que se edificó en los participantes al ser parte de CEAFRO. No se trata de un marco teórico, por ello no se concluye o se escoge cual es la utilizada para el análisis. Se entiende como perspectivas teóricas, todas útiles para reflexionar la experiencia de los integrantes del colectivo.

\section{Metodología}

La investigación se plantea siguiendo los fundamentos epistemológicos del enfoque socio-crítico, pues es desde dicho enfoque en donde mejor se puede dar respuesta a la pretensión de comprender en profundidad las significaciones, relaciones y atribuciones de la práctica participativa (Granja y Vitón, 2018), construidas desde la experiencia de los integrantes de la organización. En consonancia con lo anterior, la metodología desarrollada para dar cuenta de la experiencia de los participantes en el grupo de estudiantes del colectivo afrodescendiente se sitúa en la cualitativa, desde la perspectiva fenomenológica. Es así, como es posible estudiar las experiencias para contribuir en el proceso de mejora de las acciones de inclusión de la población afrodescendiente en los marcos institucionales de Educación Superior. 
La entrevista colectiva semiestructurada, fue la principal técnica de recopilación de información. Entendida como una conversación que se sostiene sobre aspectos de la vida cotidiana que, mediante la interacción entre el entrevistado y el entrevistador, es posible moverse en el tiempo para conocer los puntos de vista de los protagonistas sobre las acciones en que han estado inmersos, ya sea en calidad de implicados u observadores (Valles, 1999).

Se plantearon desde el instrumento 8 preguntas, pero al ser semiestructurada, surgieron nuevas interrogantes según la dinámica de la conversación. Las preguntas giraron en torno a la forma como se vinculan al colectivo, tiempo en el mismo, motivaciones para pertenecer a él, así como beneficios obtenidos. En total se entrevistaron a 8 personas que tuvieron la posibilidad de participar, de las 10 que hacen parte del núcleo dinamizador del colectivo CEAFRO. Las dos restantes por motivos personales no pudieron atender la invitación.

También se utilizó la encuesta y el análisis de bases de datos de la institución para dar cuenta de la dimensión cuantitativa en aspectos demográficos de la población estudiantil. La encuesta, se realizó con la técnica de muestreo no probabilístico por conveniencia, la cual permite seleccionar los casos que acepten ser encuestados (Otzen y Manterola, 2017); para el caso de la investigación, el criterio para aplicarla fue estudiantes que se reconocen como afrodescendientes y que quisieran libremente contestar las preguntas. Las encuestas fueron totalmente anónima.

Con dichas encuestas, se obtuvieron datos que permitieron caracterizar a los estudiantes en torno a variables como el estrato socioeconómico, lugar de procedencia, aspectos sociales, culturales y sobre la existencia de discrimancion, entre otros aspectos. En total se realizaron 8 preguntas a un total de 211 estudiantes, de una población total de 2.055 personas que se autoreconocen con afrodescendientes, según la base de datos de estudiantes de la Universidad Santiago de

\section{Cali en Colombia.}

En coherencia con el enfoque socio crítico $\mathrm{y}$, considerando el ámbito de trabajo educativo donde se inscribe, la reflexión pedagógica crítica es clave para reconsiderar este ejercicio de aprendizaje como un proceso emancipatorio (Granja y Cano, 2017). Así, dicho proceso significa que los integrantes y participantes de los colectivos intercambian ideas y sus visiones de la realidad, entre otras situaciones, y en medio de ello, surge un conocimiento creciente sobre la situación que comparten.

A través de las interacciones que se entretejen entre los participantes, se edifica una educación social no institucionalizada, que busca principalmente el surgimiento de una conciencia critica que conlleve a una praxis transformadora de la realidad (Ortega, 1999). Las entrevistas individuales, dan cuenta del significado de dichas relaciones sociales que se construyen al interior del colectivo. De esta manera, el análisis de las experiencias cotidianas, saberes y vivencias, se priorizan para desde una educación para la vida y en la vida, ir generando una apropiación crítica de las condiciones, posiciones y opciones de las personas en sus espacios institucionales.

\section{Resultados y discusión}

La ciudad de Cali, en calidad de contexto de la investigación, es una de las primeras ciudades en Colombia y América Latina, con mayor presencia de población afrodescendiente. Dicha población se caracteriza por conservar alto indice de pobreza, desempleo, y bajo cubrimiento de los servicios de salud; es una población que tiene altos índices de deserción en todos los niveles de educación.

En sintonía con lo anterior, la Universidad Santiago de Cali se caracteriza por la significativa presencia de poblacion estudiantil afrodescediente, en el 2018 cerca del $14 \%$ de sus estudiantes se consideran afrodescendientes, en su mayoría pertenecientes a estratos socioeconómicos ${ }^{(2)} 1$, 
2 y 3; dichos estratos corresponden a sectores de la ciudad y geografía nacional en donde se ubican los más bajos indicadores sociales y económicos, es decir, son estudiantes que no se caracterizan por poseer una situación económica alta y ello configura uno de los aspectos fuertes para la deserción estudiantil. Inclusive, investigaciones recientes han evidenciado la influencia que puede tener el estrato socioeconómico en la comprensión lectora de los estudiantes (Cortes, Daza y Castañeda, 2019; Cortes, Castañeda y Daza, 2019).

Según los resultados de la encuesta aplicada en el marco de la investigación realizada $\mathrm{y}$, el análisis de la base de datos institucional suministrada por la Universidad, a continuación se describen sus características más relevantes. En consecuencia se puede decir, que más del $40 \%$ de los y las estudiantes no son oriundos de la ciudad de Cali (ver Gráfico I). En tal sentido, son personas solas o con sus familiares, que han migrado a la ciudad para cumplir con sus sueños de ser profesionales.

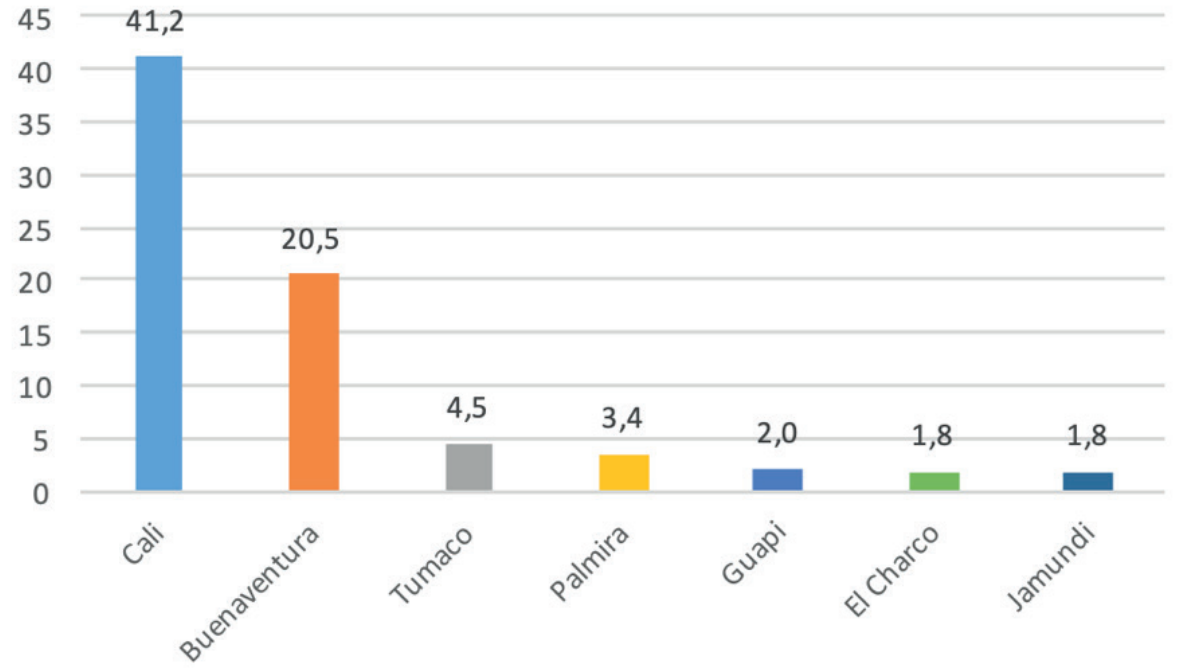

Fuente: Elaboración propia, 2020, a partir de datos de la Universidad Santiago de Cali (2008).

Gráfico I: Municipio de origen de los estudiantes afrodescendientes

En cuanto a lo social, se observa que los estudiantes reconocen la existencia de la discriminación racial en la ciudad y que ello tiene implicaciones en lo laboral, en las relaciones sociales e inclusive en lo educativo, pues muchas veces, la mirada de inferioridad que hay hacia ellos y ellas, hace que no se valoren las competencias y capacidades para desempeñarse en ciertos ámbitos laborales y profesionales.

En lo cultural, son estudiantes que se identifican politicamente como 
afrodescendientes o negros, la mayoría, el $75 \%$ de ellos/as tienen conocimiento sobre los aspectos culturales más representativos de dicha población, como lo son las danzas y los cantos. Sin embargo, poco se conoce sobre otras prácticas culturales como rittuales de nacimiento y/o de muerte. Lo anterior, se pude explicar en gran parte porque los medios de comunicación han divulgado fuertemente eventos culturales y artísticos como el "Petronio Alavarez" pero, otros eventos no han sido tan mediáticos, por ejemplo, aquellos centrados en la tradición oral. Ello hace que muchas personas crean que el sello más importante de la cultura afrocolombiana sean las danzas y los cantos.

En este contexto es donde el CEAFRO desarrolla sus actividades académicas, culturales e investigativas. El colectivo surge en el año 2008. En la revisión documental al respecto, se encuentra el proyecto de creación del Centro, en dicho documento se manifiesta como problema central que debe atender lo siguiente:

La problemática de exclusión, discriminación, pobreza corresponden a elementos neutrales que deben estudiarse desde el contexto de la cultura Afro. De igual forma, generar alternativas, herramientas y estrategias de erradicación de ciertas problemáticas que afectan a la mayoría de la población en estado de desigualdad y de marginalidad. Los problemas de una comunidad étnica se resuelven cuando se interviene desde el ámbito cultural, por ello, la comunidad de estudios Afrocolombianos CEAFRO, propondrá estudios que reorienten las políticas públicas desde una mirada investigativa. (Universidad Santiago de Cali, 2008, p.5)

Ante dicha problemática se justifica la creación de CEAFRO, articulado institucionalmente al Departamento de Humanidades. En sus 13 años de existencia, han pasado un gran número de estudiantes aportando a su funcionamiento, al igual que también la organización seguramente ha impactado en la mirada de la población afrodescendiente.

\subsection{La experiencia en CEAFRO}

Las organizaciones afrodescendientes al interior de las instituciones de educación superior, contribuyen en mejorar las condiciones sociales y académicas de los participantes, tal vez el aporte más significativo está en que coadyuva en la construcción de universidades inclusivas y respetuosas de la diferencia. En el caso particular del colectivo CEAFRO, se puede decir que busca además de lo anterior, que los estudiantes afrodescendientes no abandonen la educación superior, fortalecer los lazos identitarios, apoyar fraternal y emocional sus integrantes. En tal sentido, es una organización importante para el mejoramiento de las condiciones de la población estudiantil afrodescendiente. Ella se convierte en la materialización de políticas de inclusión.

Al respecto, las áreas de desarrollo para los estudiantes se pueden sintetizar en diferentes aspectos: Académico, social, cultural y económico. El aspecto académico, hace referencia a los apoyos colaborativos entre los mismos estudiantes afrodescendientes, en torno a algunas asignaturas que resultan complejas de comprender, por ejemplo, las matemáticas y estrategias de investigación. De igual forma, el círculo de estudio sobre historia afro, constituyen actividades extracurriculares impulsadas por el colectivo.

El aspecto social, resulta ser fundamental para el estudiante afrodescendiente, pues muchos de ellos vienen de otros municipios o de las zonas rurales y les cuesta un poco comprender las lógicas de la ciudad y la universidad, lo cual impacta en su relacionamiento social conllevando que no se generen rápidamente redes sociales para el desarrollo de la vida cotidiana.

En el aspecto cultural, en consonancia con lo social, se realizan actividades de integración, pero también de reconocimiento étnico y de valoración de las costumbres ancestrales. Por su parte, el aspecto económico, tiene varias actividades, como, por ejemplo; charlas de emprendimiento afrodescendiente, gestionar y brindar información sobre las becas 
condonables del ICETEX, las cuales resultan de ayuda económica para que los estudiantes puedan culminar sus estudios.

Todo lo anterior, conlleva a que los estudiantes se adapten a la vida universitaria y construyan su vida social a través de la cotidianidad que se comparte con otros estudiantes afrodescendientes. De igual forma, asuman una actitud frente a la vida de manera crítica, propositiva y con responsabilidad social, en términos de ayudar o contribuir que más personas afrodescendientes puedan salir adelante mejorando su calidad de vida.

A partir de la entrevista y del marco teórico, es posible reflexionar porqué el colectivo se mantiene cohesionado y realizando las actividades anteriormente mencionadas, las cuales, como se indica líneas arriba, permiten que los integrantes no deserten de la educación superior, y por el contrario, trabajen por el bienestar de los demás estudiantes favoreciendo la inclusión.

Con el fin de identificar las razones de lo anterior, en la investigación, se determinaron las categorías de análisis que surgieron, es decir, a través de los relatos -de la entrevista colectiva- se identifican los aspectos en común y el significado que tiene para los integrantes de CEAFRO y, que pueden encontrar un dialogo con lo expuesto en el marco teórico. De tal suerte que, lo manifestado por los participantes se codificó teniendo el lugar común de significación. Como resultado de dicho ejercicio, surgieron las siguientes categorías de análisis (ver Cuadro 1), que permiten explicar por qué siguen en el colectivo y con ello, continúan en la universidad realizando sus estudios.

\section{Cuadro 1}

\section{Categorías de análisis y su incidencia en la participación}

\begin{tabular}{|c|c|c|}
\hline Categorías de Análisis & Relación con las teorías & $\begin{array}{l}\text { Significado a partir de lo expresado } \\
\text { por los integrantes de CEAFRO }\end{array}$ \\
\hline $\begin{array}{l}\text { IDENTIDAD Y } \\
\text { CONCIENCIA }\end{array}$ & $\begin{array}{l}\text { Hace a referencia a factores identitarios; } \\
\text { como son las visiones compartidas, inclusive } \\
\text { culturalmente, metas comunes por los } \\
\text { integrantes, entre otros aspectos, que hacen } \\
\text { surgir un marco de significación de la realidad } \\
\text { colectiva. dos de los autores que se citan en esta } \\
\text { perspectiva son: Shaw (2013) y Torres (2007). }\end{array}$ & $\begin{array}{l}\text { Alineación entre la visión e intereses } \\
\text { personales con los colectivos. La } \\
\text { situación de discriminación étnica, el } \\
\text { compartir historias comunes y coincidir } \\
\text { en las apreciaciones sobre la realidad } \\
\text { vivida hace que exista identidad entre los } \\
\text { integrantes de CEAFRO y, conciencia } \\
\text { sobre que se debe salir adelante a pesar } \\
\text { de las adversidades. }\end{array}$ \\
\hline PERTENENCIA & $\begin{array}{l}\text { Se refiere a las condiciones socio-afectivas que } \\
\text { se establecen en la interacción con los otros y, } \\
\text { que, hacen surgir el sentimiento de fraternidad, } \\
\text { de ser parte de algo Uno de los autores que se } \\
\text { citan en esta perspectiva es Lewin (1978). }\end{array}$ & $\begin{array}{l}\text { El sentir que se hace parte de un grupo, y } \\
\text { que todos/as se consideran con parte de } \\
\text { una familia, hace que surjan sentimientos } \\
\text { de hermandad, que se revierten en apoyo } \\
\text { mutuo. }\end{array}$ \\
\hline
\end{tabular}

Fuente: Elaboración propia, 2020.

En consonancia con el Cuadro anterior, se puede mencionar que CEAFRO se constituye para sus integrantes en una red de apoyo, de solidaridad, en donde el estudiante encuentra otra familia, un grupo de amigos y espacios para compartir su tiempo libre. En palabras de uno de los integrantes del colectivo, se menciona lo que significa CEAFRO: "Es un lugar donde aprendemos otras cosas diferentes a lo de nuestras carreras, aprendemos y 
nos concientizamos sobre la situación de los negros y como se da la discriminación" (Entrevista a integrante de CEAFRO, 2018). Otra entrevista sitúa a CEAFRO como espacio de fortalecimiento de redes sociales. "Para mi CEAFRO es como mi familia compartimos, aprendemos y nos ayudarnos unos a otros. Nos aceptamos como somos y eso me llena de orgullo" (Entrevista a integrante de CEAFRO, 2018).

Las anteriores apreciaciones pueden ser explicadas desde la perspectiva del campo desarrollada por Lewin (1978), pues, las expresiones de los estudiantes hacen referencia al sentimiento fraternidad e identidad hacia el colectivo y con ello su pertenencia hacia el mismo. Para los integrantes de CEAFRO, resulta de gran importancia las redes sociales y espacios académicos extracurriculares en el afianzamiento y formación académica de los estudiantes, en especial, para la población afrodescendiente.

De igual forma, es clara la incidencia del sentimiento de pertenencia en la participación y por supuesto en la identidad. La conformación de la identidad a partir de lo afectivo, es una de las ideas en el profesor Torres (2007), al argumentar que la identidad colectiva requiere -además de otros factores- de la solidaridad, los vínculos afectivos y la ideología que maneja el grupo o la organización. Sin embargo, esto no es posible sin la existencia de la convergencia entre los intereses y necesidades individuales con los colectivos, a tal punto que a veces para el integrante es difícil jerarquizar sus compromisos personales con los colectivos, siendo esta una de las muchas formas de evidenciar el sentido de pertenencia y entrega a las acciones colectivas que más caracterizan a los integrantes del colectivo, lo cual es evidenciado en los diferentes apartes de las entrevistas realizadas, a manera de ilustración se transcriben algunas de ellas:

CEAFRO es como mi familia, paso mucho tiempo con mis compañeros de la organización, ellos ocupan ese espacio familiar dado que no soy de Cali, por lo tanto, ellos son muy valiosos para mí. Entonces yo sí creo que existe un apego, mucho cariño y ello hace que yo tenga identidad hacia el grupo CEAFRO. (Entrevista a integrante de CEAFRO, 2018).

No hay un día mejor para mi en la universidad que cuando citamos a reunión, o nos encontramos para planear o desarrollar alguna actividad. Son días que están atravesados por mucha afectividad, no somos amigos, somos hermanos $y$ hermanas y nos apoyamos en los casos cuando a alguien le va mal o ha sufrido algún momento de discriminación. No damos apoyo mutuo y nos identificamos en nuestra historia y experiencias. (Entrevista a integrante de CEAFRO, 2018).

Aunque estos son sólo dos de los relatos que demuestran esa conectividad sentimental o de afectividad hacia el grupo, es importante mencionar que los demás integrantes del CEAFRO muestran una actitud similar. Lo trascendental para futuros análisis de organizaciones, es tomar la afectividad que genera la pertenencia o viceversa, como elemento fundamental de estudio. Entendida esta como el aprecio, amor o cariño que comienza a fluir entre los integrantes de una colectividad y, que, en buena medida, determina y permite explicar porqué las personas más allá de los intereses compartidos y a pesar de los conflictos y malentendidos que no faltan, tomen la determinación de seguir juntos. Lo anterior puede ser explicado a partir de la teoría de la interacción desde la perspectiva de Shaw (2013); la existencia de necesidades comunes hace que los colectivos se cohesionen.

El anterior elemento, permea el significado de las acciones que se realizan en el marco de la organización, pues hace que no sean desprovistas del significado sentimental que adquieren en donde corren cierta suerte en su mayoría de ser altruistas, dado que por ellas ninguno de los integrantes recibe una retribución económica más allá del placer de realizarlas. Aunque seguramente reciben a cambio aprecio, reconocimiento, la construcción de un sentido de vida, lazos de solidaridad, y sobre todo satisfacción con lo que hacen. Lo anterior podría asociarse con las necesidades humanas explicadas por 
Maslow (1991), particularmente aquellas que se relacionan con las de pertenencia y de estima. No obstante, lo que subyace a dichas necesidades puede verse referenciado en una búsqueda de combatir la soledad, los sentimientos de alienación y de desesperanza

\section{Conclusiones}

Es recomendable que las instituciones de educación superior trabajen operativamente en construir un ambiente de inclusión a los grupos afrodescendientes, en donde no solo existan acciones que se concreten en la ampliación de cupos en la diferentes carreras, sino que también respondan a las necesidades de formación que demandan las comunidades de la población afrodescendiente; igualmente fomentar y desarrollar políticas de adaptación cultural hacia la vida universitaria, que busquen minimizar el choque cultural o social de los estudiantes, principalmente aquellos que vienen de otras regiones.

Asimismo, buscar medios alternativos de subsidios económicos para financiar y garantizar que aquellas personas que no cuenten con suficientes recursos económicos, para atender sus necesidades propias que demanda estar en la educación superior, puedan culminar sus estudios, asegurando de esta forma que alcancen dicha meta. Revisar sus currículos y las estrategias pedagógicas de los docentes, con el fin de brindar una educación con calidad, pertinente a las necesidades de los pueblos afrodescendientes, en donde se tenga muy en cuenta sus tradiciones culturales y formas de ver la realidad.

En línea con lo anterior, los colectivos afrodescendientes deben tenerse en cuenta para el diseño de dichas políticas, pues son ellos los que conocen de primera mano las problemáticas que se presentan en la cotidianidad y su contacto permanente con la población que representan, hace que se conviertan en unos actores valiosos en la construcción de acciones desde las bases.

Al mismo tiempo, dichos colectivos son espacios ideales para desarrollar actividades de bienestar institucional, de extensión o proyección social e inclusive de investigaciones que propendan por la mejora de la calidad de vida, es decir, permiten una mejor articulación de las universidades con el medio social.

Además de las posibilidades que brindan dichas organizaciones, hay que reconocer la importante función que cumplen como protectoras y generadoras de relaciones sociales para las personas discriminadas, para aquellas que vienen desde lejos, que no tienen familias o amigos(as) -entre otras situaciones-, las cuales las convierten en organizaciones de apoyo: Un "colchón social" donde se puede encontrar lazos de solidaridad y de fraternidad. Por todo lo anterior, las universidades deben fomentarlas, apoyarlas, también, por toda la importancia que ellas representan en la construcción de una vida universitaria más incluyente y multicultural.

Por último, mencionar lo importante que fue para los participantes del colectivo reflexionar a través de la entrevista, la experiencia de participar en el colectivo afrodescendiente. Manifiestan con sus palabras, que nunca se habían detenido a pensar porqué siguen asistiendo al colectivo, qué es lo que más les gusta y cómo ello ha contribuido para mantenerse en la Universidad. En consecuencia, se puede decir, que la investigación fue un espacio para que se conocieran mejor, y apreciaran más la organización. En ese sentido, fue una experiencia pedagógica y transformadora, dado que reflexionaron sobre lo que son $\mathrm{y}$ hasta dónde quieren llegar.

\section{Notas}

${ }^{1}$ Entidad estatal que promueve la Educación Superior a través del otorgamiento de créditos educativos y su recaudo (ICETEX, 2019).

2 En Colombia se definen seis estratos socioeconómicos, esta clasificación la da el gobierno colombiano a los niveles socioeconómicos de la sociedad y fundamentan en los ingresos que se reciben un hogar, la 
Inclusión social de la población estudiantil afrodescendiente: Experiencia de un colectivo de estudiantes universitarios

manera en la que está construida una vivienda, el sector, las vías públicas, los servicios públicos. Según el Departamento de Planeación Nacional, los estratos socioeconómicos en los que se clasifican los sectores en donde viven los colombianos son: 1: Bajo-bajo, 2: Bajo, 3: Medio-bajo, 4: Medio, 5: Medio-alto y 6-Alto (DANE, 2015).

\section{Referencias bibliográficas}

Barbary, O., y Urrea, F. (Eds.) (2004). Gente negra en Colombia: Dinámicas sociopolíticas en Cali y el Pacifico. Editorial Lelon.

Chenet, M. E., Arévalo, J. C., y Palma, F. (2017). Identidad cultural y desempeño docente en instituciones educativas. Revista Opción, 33(88), 292-322.

ConsejoNacionaldeAcreditación-CNA(2013). Lineamientos para la acreditación de programas de pregrado. SECAB-PUBLICACIONES. https:// www.mineducacion.gov.co/1621/ articles-342684 recurso 1.pdf

Cortes, J., Castañeda, J., y Daza J., (2019). Comprensión lectora de estudiantes universitarios. Factores asociados y mecanismos de acción. Revista Venezolana de Gerencia, 24(87), 874889.

Cortes, J. E., Daza, J., y Castañeda, J. G. (2019). Relación del entorno socioeconómico con el desempeño de la comprensión lectora en universitarios. Revista de Ciencias Sociales (Ve), XXV(4), 119133.

De Sousa, B. (2007). La Universidad en eL sigLo XXI. Para una reforma democrática y emancipatoria de la universidad. Plural Editores.

Departamento Administrativo Nacional de Estadística - DANE (2019). Población negra, afrocolombiana, raizal $y$ palenquera: Resultados del censo nacional de población y vivienda 2018. DANE. https://bit.ly/333p6oD

Díez, E. (1999). La estrategía del caracol: El cambio cultural en una organización. Oikos-Tau, S.A. Ediciones.

Fernández, C. (2009). La comunicación en las organizaciones. Trillas.

García, I., Fletcher, T., y Romero, S. (2009). Avances y retos de la Educación Inclusiva en Latinoamérica. En M. A. Casanova y H. Rodríguez (Coords.), La inclusión educativa, un horizonte de posibilidades (pp. 225-272). La Muralla.

Granja, L. C., y Cano, M. C. (2017). La investigación formativa como estrategia pedagógica para la construcción del conocimiento. Una mirada desde las políticas, los actores, las prácticas y los discursos. En M. C. Cano (Comp.), El prisma de la formación docente en Colombia. Teoría pedagógica y experiencias didácticas (pp. 53-67). Editorial Universidad Santiago de Cali.

Granja, L. C., y Vitón, M. J. (2018). Empoderamiento organizativo afrodescendiente: Una experiencia de investigación acción participativa. Editorial Académica Española.

Instituto Colombiano de Crédito Educativo y Estudios Técnicos en el Exterior ICETEX (2019). Introducción. https:// portal.icetex.gov.co/Portal/Home/elicetex/quienes-somos/introducción

Lewin, K. (1978). La teoría del campo en la ciencia social. Paidós Iberica.

Martin-Fiorino, V. (2020). Responsabilidad social y cultura de la integridad: Formación de profesionales para la sostenibilidad. Revista de Ciencias Sociales (Ve), XXVI(4), 162179. https://doi.org/10.31876/rcs. v26i4.34655

Licencia de Creative Commons Atribución 4.0 Internacional (CC BY 4.0) https://creativecommons.org/licenses/by/4.0/deed.es 
Maslow. A. H. (1991). Motivación y personalidad. Díaz de Santos.

Ministerio de Cultura y Universidad de los Andes (2009). Panorama socioeconómico y político de la población afrocolombiana, raizal y palenquera. Retos para el diseño de politicas públicas. $\underline{\text { https://bit. }}$ ly/3eGWAic

Montañés, M. (2007). Más allá del debate cuantitativo/cualitativo: La necesidad de aplicar metodologías participativas conversacionales. Revista Politica y Sociedad, 44(1), 13-29.

Montero, M. (2003). Teoría y práctica de la psicología comunitaria: La tensión entre comunidad y sociedad. Editorial Paidós.

Nuñez, L. A., Lescano, G. S., Ibarguen., F. E., y Huamani, L. N. (2019). Consideraciones teóricas en torno a la Responsabilidad Social de la Educación. Revista Venezolana de Gerencia, 24(87), 725-735.

Organización de Naciones Unidas - ONU (2020). Objetivos de Desarrollo Sostenible. Objetivo 4: Garantizar una educación inclusiva, equitativa y de calidad y promover oportunidades de aprendizaje durante toda la vida para todos. ONU. http://www.un.org/ sustainabledevelopment/es/education/

Organización para la Cooperación y el Desarrollo Económicos - OCDE (2011). Perspectives on Global Development 2012: Social Cohesion in a Shifting World. https://www.oecd. org/dev/pgd/perspectivesonglobaldev elopment2012socialcohesioninashiftin gworld.htm

Ortega, J. (Coord.) (1999). Educación social especializada: Educacion con menores en dificultad $y$ en conflicto. Editorial Ariel, S.A.

Otzen, T., y Manterola, C. (2017). Técnicas de muestreo sobre una población a estudio. International Journal of Morphology, 35(1), 227-232. http://dx.doi.org/10.4067/S0717$\underline{95022017000100037}$

Red Universitaria Mutis (2015). Aportes para una política de acciones afirmativas en Colombia/guía para universidades. Red Mutis.

Rodríguez, C. A., Alfonso, T., y Cavelier, I. (2008). El derecho a no ser discriminado: Primer informe sobre discriminación racial $y$ derechos de la población afrocolombiana. Universidad de los Andes. Observatorio de Discriminación Racial.

Rubio, G. A., Estrada, J. M., y Pedraza, G. (2020). Responsabilidad social universitaria: Incidencia en diferentes grupos de interés en una universidad colombiana. Revista de Ciencias Sociales (Ve), XXVI(4), 180189. https://doi.org/10.31876/rcs. v26i4.34656

Shaw, M. (2013). Dinámica de grupo: Psicología de la conducta de los pequeños grupos. Herder Editorial.

Torres, A. (2007). Identidad y politica de la acción colectiva. Nomos.

Universidad Santiago de Cali (2008). Colectivo de Estudios Afrocolombiano. Universidad Santiago de Cali (Colombia).

Valles, M. (1999). Técnicas cualitativas de investigación social: Reflexion metodologica y práctica profesional. Síntesis Sociologia. 